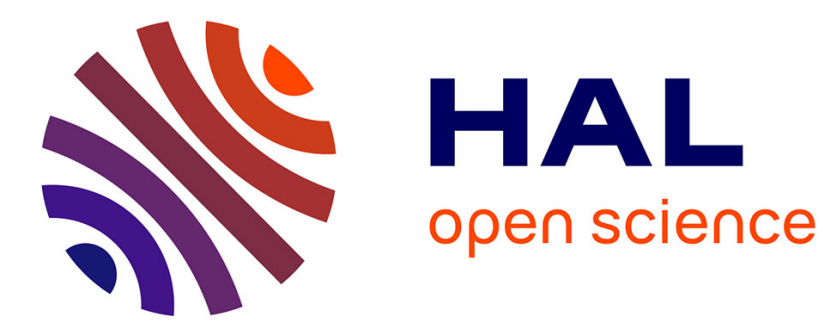

\title{
Pâturage pour vaches laitières. Effets du chargement et de la complémentation en pâturage tournant simplifié
}

\author{
A. Hoden, A. Müller, J.L. Peyraud, Luc Delaby, Philippe Faverdin, Jr. \\ Peccatte, M. Fargetton
}

\section{- To cite this version:}

A. Hoden, A. Müller, J.L. Peyraud, Luc Delaby, Philippe Faverdin, et al.. Pâturage pour vaches laitières. Effets du chargement et de la complémentation en pâturage tournant simplifié. Productions Animales, 1991, 4 (3), pp.229-239. hal-00895942

\section{HAL Id: hal-00895942 \\ https://hal.science/hal-00895942}

Submitted on 1 Jan 1991

HAL is a multi-disciplinary open access archive for the deposit and dissemination of scientific research documents, whether they are published or not. The documents may come from teaching and research institutions in France or abroad, or from public or private research centers.
L'archive ouverte pluridisciplinaire HAL, est destinée au dépôt et à la diffusion de documents scientifiques de niveau recherche, publiés ou non, émanant des établissements d'enseignement et de recherche français ou étrangers, des laboratoires publics ou privés. 
INRA Prod. Anim., 1991, 4 (3), $229-239$
A. HODEN, A. MULLER (1),

J.L. PEYRAUD, L. DELABY,

Ph. FAVERDIN avec la collaboration

de JR. PECCATTE (1),

et M. FARGETTON (1)

INRA, Station de Recherches sur la Vache Laitière

35590 Saint-Gilles

(1) INRA, Service d'expérimentation animale - Le Pin au Haras

61310 Exmes
Pâturage pour vaches laitières. Effets du chargement et de la complémentation en pâturage tournant simplifié

Les techniques de pâturage peuvent, sous certaines conditions, être simplifiées sans pénaliser les performances des vaches laitières. Par ailleurs, lorsque l'herbe pâturée est de bonne qualité, l'apport d'aliment concentré est en moyenne peu efficace.

En pâturage tournant simplifié, l'accroissement du chargement peut-il permettre d'améliorer les performances à l'hectare sans trop pénaliser les productions individuelles et d'augmenter l'efficacité de la complémentation en aliment concentré ?

Les systèmes d'exploitation du pâturage ont fait l'objet de nombreuses comparaisons (revues de Journet et Demarquilly 1979; de Leaver 1985 ; Hoden et al 1986 b, c). Les résultats indiquent que la simplification des méthodes de conduite à l'herbe a peu d'influence sur les performances lorsqu'elle est pratiquée selon les particularités de chaque système (Holmes 1989 b). Ainsi, la technique du pâturage tournant simplifié peut être appliquée aux vaches laitières avec un nombre de parcelles variant de 2-3 au printemps à 4-6 en fin de saison dans toutes les zones à pluviométrie

\section{Résumé}

Trois essais, chacun d'une durée moyenne de 6 mois, ont été répétés de 1986 à 1988 sur les mêmes surfaces du domaine INRA du Pin au Haras selon la technique du pâturage tournant simplifié ( 2 parcelles au printemps, 4 en automne). Ils ont concerné un total de $\mathbf{1 6 2}$ vaches d'un haut niveau de production à la mise à l'herbe $(30,0 \mathrm{~kg} / \mathrm{j}$ de lait en moyenne). Les comparaisons ont porté sur l'influence de 3 niveaux de chargement correspondant à 100 (Témoin $=2,3$ vaches/ ha), 115 (Fort $=2,6$ vaches $/$ ha) et 130 (Très fort $=3,0$ vaches $/$ ha) $\%$ du témoin, en interaction avec 2 niveaux $\mathrm{H}$ (Haut $=3,7 \mathrm{~kg} / \mathrm{j} /$ vache) et $\mathrm{B}$ (Bas $=0,5 \mathrm{~kg}$ ) d'aliment concentré. Les productions individuelles ne sont pas significativement différentes entre chargements et n'ont diminué que de $5 \%$ entre les extrêmes $(20,3$ pour le Très Fort contre $21,3 \mathrm{~kg}$ lait $4 \%$ pour le Témoin). La production de lait à l'hectare a augmenté de $23 \%$ avec le chargement Très Fort ( 9800 contre environ $8000 \mathrm{~kg}$ lait $4 \%$ avec le Témoin). La complémentation a permis une amélioration moyenne des productions de $9 \%(21,8$ avec le niveau Haut contre $20,0 \mathrm{~kg}$ lait $4 \%$ avec le Bas $P<0,01)$. Cette réponse tend à s'améliorer avec le niveau de production des vaches à la mise à l'herbe et l'augmentation du chargement. Les mesures effectuées sur l'herbe pâturée permettent de mieux appréhender sa valorisation par les vaches. bien répartie permettant de valoriser des apports azotés annuels de 250 à $300 \mathrm{~kg}$ par ha. Dans ces conditions, à mêmes niveaux de chargement et de complémentation, les performances individuelles et à l'hectare sont comparables à celles obtenues avec le pâturage rationné (Hoden et Muller 1989).

Cependant les variations du niveau des apports nutritifs à l'herbe, par l'intermédiaire du chargement et de la complémentation, ont rarement été étudiées avec la technique du pâturage tournant simplifié. Il importe pourtant de savoir si l'accroissement du chargement, tout au moins jusqu'à un point critique, modifie peu les performances individuelles, occasionnant de ce fait une augmentation importante des performances globales à l'hectare (Mott 1960 ; Mayne et al 1987). De même, il est simultanément nécessaire de savoir si l'efficacité de la complémentation à l'herbe reste faible (revue de Leaver 1985 ; Hoden et al 1987 ; Kibon et Holmes 1987) même dans le cas de chargements élevés et avec des vaches de bon potentiel.

Ce thème a été abordé durant 3 saisons consécutives au domaine INRA du Pin au Haras (Normandie) en faisant varier le niveau de chargement en interaction avec la complémentation aux alentours des recommandations habituellement pratiquées. L'objectif était de mesurer, sur l'ensemble de la saison, les effets de tels traitements sur les performances au pâturage dans le cas de vaches à haut niveau de production à la mise à l'herbe. Ces études devaient aussi nous permettre d'analyser les 
variations des productions durant l'exploitation des parcelles et, d'avoir une meilleure approche explicative des écarts de performances par l'intermédiaire de différents critères mesurés à la fois sur l'animal et le couvert végétal.

\section{Conditions expérimentales}

\section{1 / Traitements}

Un schéma expérimental factoriel combinant 3 chargements à 2 niveaux de complémentation a été utilisé. Les 3 chargements ont été dénommés Témoin $(\mathrm{T})$, Fort $(\mathrm{F})$ et Très Fort $(\mathrm{TF})$. Le chargement $\mathrm{T}$ était celui habituellement pratiqué dans les conditions du Pin au Haras, soit 4,7 vaches par hectare au printemps et 2,3 en automne. Les traitements $F$ et $\mathrm{TF}$ correspondaient à des niveaux de 115 et $130 \%$ respectivement du chargement $\mathrm{T}$. Les deux niveaux de complémentation ont été désignés par Haut $(\mathrm{H})$ et Bas (B). Le niveau $\mathrm{H}$ correspondait à un apport individuel et constant sur l'ensemble de la saison d'un aliment concentré $10,95 \mathrm{UFL} / \mathrm{kg}$ et $120 \mathrm{~g} \mathrm{PDI/UFL} \mathrm{selon} \mathrm{INRA} \mathrm{1988)} \mathrm{distribué} \mathrm{à}$ raison de $330 \mathrm{~g}$ par $\mathrm{kg}$ de lait au dessus de $20 \mathrm{~kg}$ lors de la mise à l'herbe. Les vaches du niveau $B$ n'ont reçu qu'une complémentation constante de $500 \mathrm{~g} / \mathrm{jour}$ d'un aliment permettant l'apport de $200 \mathrm{~g}$ de minéraux (12 P - $16 \mathrm{Ca}$ - $5 \mathrm{Mg}$ ) + vitamines. En moyenne, les vaches du niveau $\mathrm{H}$ ont consommé $3,2 \mathrm{~kg} /$ jour d'aliment concentré supplémentaire par rapport au niveau B compte tenu de la complémentation minérale systématique.

L'expérimentation a duré 3 ans (1986, 1987 et 1988) sur une période d'environ 6 mois d'avril à octobre. Chaque année, à partir du troupeau de 80 Holstein et Normandes $(2 / 3$, $1 / 3$ respectivement), 54 vaches ont été réparties en 6 groupes de 9 vaches appariées suite à une période préexpérimentale de 3 semaines située

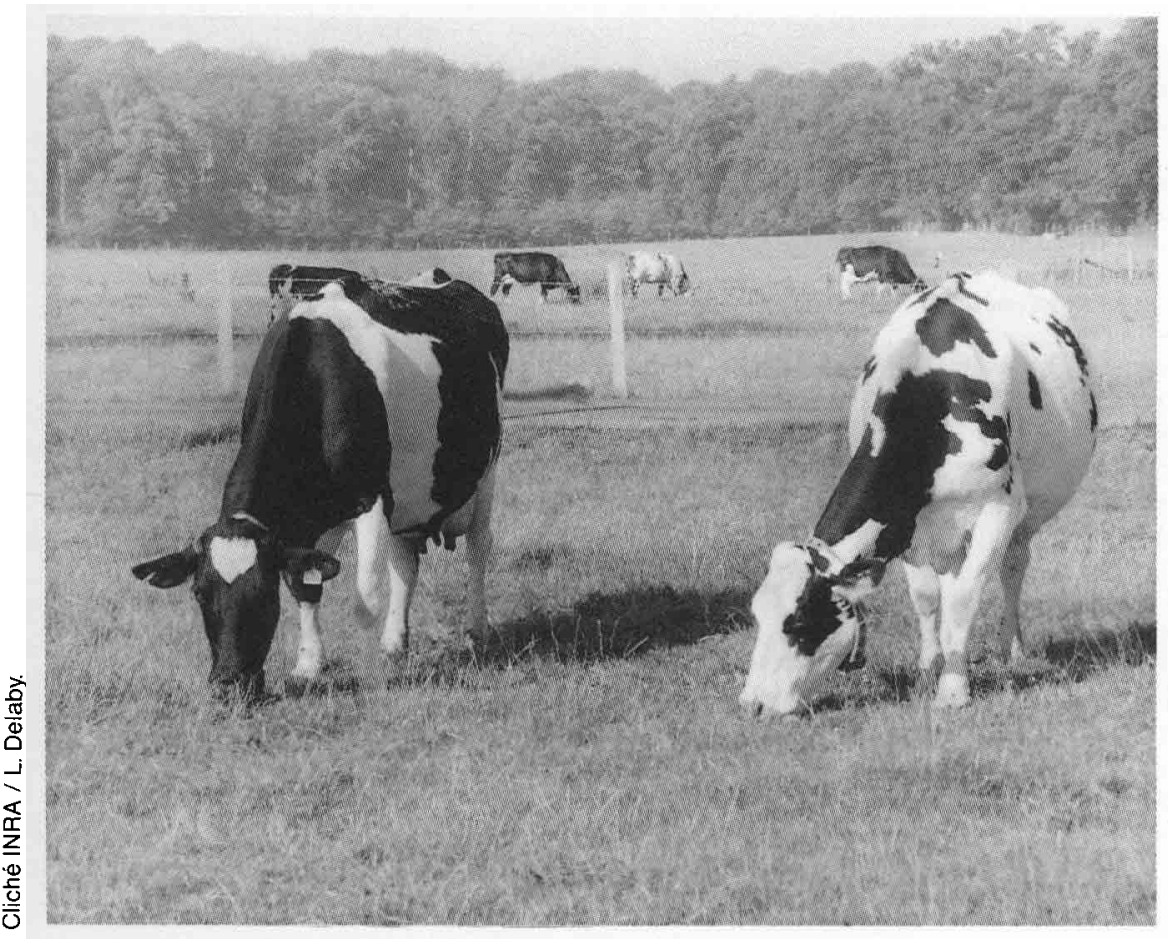

lors de la mise à l'herbe. Les critères ont été le numéro de lactation ( $27 \%$ de primipares), la date du vêlage ( 9 février $\pm 29 \mathrm{j}$ ), la production de lait $(30,2 \pm 5,9 \mathrm{~kg})$ et sa composition $(39,7$ $\pm 3,8 \mathrm{~g} / \mathrm{kg}$ de taux butyreux et $30,2 \pm 2,3 \mathrm{~g} / \mathrm{kg}$ de taux protéique) ainsi que le poids vif (670 \pm $63 \mathrm{~kg}$ ).

\section{2 / Conduite du pâturage}

La technique du pâturage tournant simplifié ( 2 parcelles au printemps, 4 en fin de saison) mise au point dans les conditions du Pin au Haras (Hoden et al 1986 b et c) a été appliquée. Pour le traitement $\mathrm{T}$, les règles de conduite du pâturage ont été dictées par les variations de la production laitière durant le temps de séjour sur chaque parcelle. En se basant sur nos précédents résultats (Hoden et Muller 1989), le changement de parcelle a eu lieu lorsque la quantité de lait avait chuté de $10 \%$ par rapport au maximum de production observé. La même date a été systématiquement appliquée aux traitements $\mathrm{F}$ et TF, quels que soient leurs niveaux de production à ce moment.

La vitesse de rotation des différents cycles a simultanément été ajustée de façon à maintenir une bonne valeur nutritive de l'herbe et à en offrir au minimum $800 \mathrm{~kg}$ de MS/ha à l'entrée sur chaque parcelle. Elle a ainsi été de 4 semaines au printemps et a atteint un maximum d'environ 6 semaines en fin de saison grâce à un ajustement du nombre de parcelles à chaque cycle.

Les surfaces de base étaient des prairies permanentes drainées constituées de ray grass anglais, de pâturin commun et d'agrostis en proportions comparables (20 à $25 \%$ ), le reste étant de la houlque laineuse $(15 \%)$ et de la fléole ( $5 \%$ ). La surface additionnelle pâturée après la récolte des excédents de printemps sous forme d'ensilage, était une prairie d'association à base de ray grass anglais et de trèfle blanc semée en avril 1984. La fertilisation azotée, réalisée en 5 épandages, était de $300 \mathrm{~kg}$ d'N/ha/an sur les parcelles pâturées; les apports de $\mathrm{P}$ et de $\mathrm{K}$ étaient de $150 \mathrm{~kg}$ tous les 2 ans.

\section{3 / Mesures et interprétation des résultats}

\section{a / Zootechniques}

Les quantités de lait produites ont été mesurées à chaque traite à l'aide de compteurs. La composition du lait (taux butyreux et protéique) de chaque vache a été déterminée sur un échantillon représentatif de 4 traites par semaine. Simultanément, cette détermination a été effectuée quotidiennement à partir d'un prélèvement global de lait correspondant à chacun des lots de 18 vaches des différents chargements. Les animaux ont été pesés à chaque début de cycle de pâturage. Les quantités d'aliments concentrés ingérées ainsi que les éventuels apports de fourrages complémentaires (ensilage de maïs) ont été pesés.

Les quantités de matière organique d'herbe ingérées ont été estimées à 5 reprises aux prin- 
temps 1987 et 1988: 3 au milieu (jours 7 à 10) et 2 en fin (jours 11 à 14) du temps de séjour sur différentes parcelles. Les mesures ont été réalisées uniquement pour les traitements $\mathrm{T}$ et TF sur les prairies permanentes. Ces quantités ont été estimées selon la méthode décrite par Peyraud et al (1989). Du concentré contenant $20 \mathrm{~g}$ d'oxyde de chrome/ $\mathrm{kg}$ a été ingéré en 2 fois par jour lors des traites durant toutes ces périodes. La digestibilité de la matière organique (DMO) a été déterminée à partir de la teneur en azote des fèces ( $\mathrm{N} \% \mathrm{MO}$ ) en utilisant l'équation suivante (Peyraud non publié) : DMO $=0,195+0,271 \times \mathrm{N}-0,0288 \times \mathrm{N}^{2}\left(\mathbf{n}=21, \mathrm{R}^{2}=\right.$ 0,85, Syx $=0,017$ ). Toutes les bouses fraîches présentes sur l'ensemble de la parcelle ont été prélevées chaque matin. Les fèces des animaux $\mathrm{B}$ et $\mathrm{H}$ ont été distinguées grâce à la distribution de $60 \mathrm{~g} / \mathrm{j}$ de particules de plastique colorées en même temps que les agglomérés d'oxyde de chrome. Pour les vaches du niveau $\mathrm{H}$, l'excrétion de fèces issue de l'herbe a été calculée en soustrayant celle due à l'aliment concentré ( $150 \mathrm{~g}$ de $\mathrm{MO}$ non digestible $/ \mathrm{kg}$ de MO d'aliment ingéré - INRA 1988) en supposant que la digestibilité du fourrage n'était pas affectée par l'aliment concentré.

\section{b / Sur le végétal}

La production d'herbe a été estimée avant l'entrée des animaux en coupant 8 bandes $(10 \mathrm{x}$ $0,5 \mathrm{~m}$ l'une) par parcelles à l'aide d'une motofaucheuse (coupe à $5 \mathrm{~cm}$ du niveau du sol). Ces prélèvements correspondent à environ $0,25 \%$ de la surface offerte aux animaux. La collecte totale des 8 bandes a été pesée, mélangée et échantillonnée en vue d'en déterminer la teneur en MS ( $48 \mathrm{~h}$ à $80^{\circ} \mathrm{C}$ ). La hauteur de l'herbe a été mesurée en 1987 et 1988 à partir d'un herbomètre muni d'un plateau carré en aluminium $\left[0,09 \mathrm{~m}^{2}\right.$ de surface - pression de 4,5 $\mathrm{kg} / \mathrm{m}^{2}$ ) en effectuant 150 mesures/ha avant l'entrée et après la sortie des animaux de chaque parcelle (Castle 1976). Chaque mesure effectuée à la sortie a été notée de façon qualitative par l'opérateur selon le degré d'utilisation de l'herbe par les animaux (zone fréquentée ou peu fréquentée). La densité brute moyenne de l'herbe ( $D=\mathrm{kg} / \mathrm{cm} / \mathrm{ha}$ ) a été calculée en effectuant le rapport entre la biomasse récoltée et la hauteur de l'herbomètre à l'entrée diminuée de $5 \mathrm{~cm}$ (hauteur de coupe de la motofaucheuse).

\section{c / Analyses statistiques et bilans}

Toutes les données ont été analysées en utilisant la procédure General Linear Model de SAS (1987). Pour les performances individuelles, les données obtenues en préexpérience ont été utilisées comme covariables. Les autres termes du modèle ont été les traitements (année, chargement, niveau de complémentation), les interactions entre traitements et les interactions de pentes significatives $(\mathrm{P}<0,05)$ entre les traitements et les covariables. Les quantités d'herbe ingérées ont été analysées selon un modèle partiellement hiérarchisé prenant en compte le chargement, la complémentation, leur interaction, le temps de séjour (milieu vs fin) et la période. Pour la digestibilité, le niveau de concentré n'a pas été considéré. Toutes les données sur le végétal (biomasse, hauteur et densité) ont aussi été analysées selon un modèle hiérarchisé en considérant les variables : année, chargement, type de prairies (permanentes vs temporaires), parcelle, cycle dans parcelle et interactions. Les bilans globaux à l'hectare ont été calculés selon la méthode INRA (Hoden et al 1986 a).

\section{Résultats}

\section{1 / Déroulement du pâturage}

Durant les 162 jours d'expérimentation annuelle, les conditions climatiques (figure 1)

Tableau 1. Caractéristiques générales du pâturage (Moyennes des 3 années).

\begin{tabular}{|lc|c|c|c|}
\hline \multicolumn{1}{|c|}{ Chargement } & & Témoin (T) & Fort (F) & Très Fort (TF) \\
\hline Chargement (vaches/ha) & PP & 4,7 & 5,3 & 6,0 \\
& ST & 2,3 & 2,6 & 3,0 \\
Effectif (1) & & - & 18 & - \\
Durée (j) (2) & PP & - & 108 & - \\
Surfaces pâturées (ha) (3) & ST & - & 162 & - \\
& PP & 3,8 & 3,4 & 3,0 \\
Nombre de jours & ST & 7,8 & 6,8 & 6,0 \\
de pâturage (j/ha) (4) & PP & 512 & 572 & 648 \\
Nombre parcelles & ST & 374 & 429 & 486 \\
Temps de séjour par parcelle (j) & & 2 au printemps $\rightarrow 4$ en été \\
Vitesse de rotation (j) & & 14 au printemps $\rightarrow 7$ en été \\
Surface fauchée Printemps (ha) & & - & 35 & - \\
Herbe récoltée (kg MS/ha ST) & & 4,8 & 4,2 & 3,7 \\
Fumure azotée (kg N/ha) & & - & 2400 & - \\
& PP & - & 254 & - \\
\hline
\end{tabular}

PP : Prairies permanentes; ST : Surface totale ; (4) = (1) $\times(2) /(3)$ 
Figure 1. Conditions climatiques mensuelles (comparaison par rapport à la moyenne 1961 - 1985).

\section{Pluviométrie}
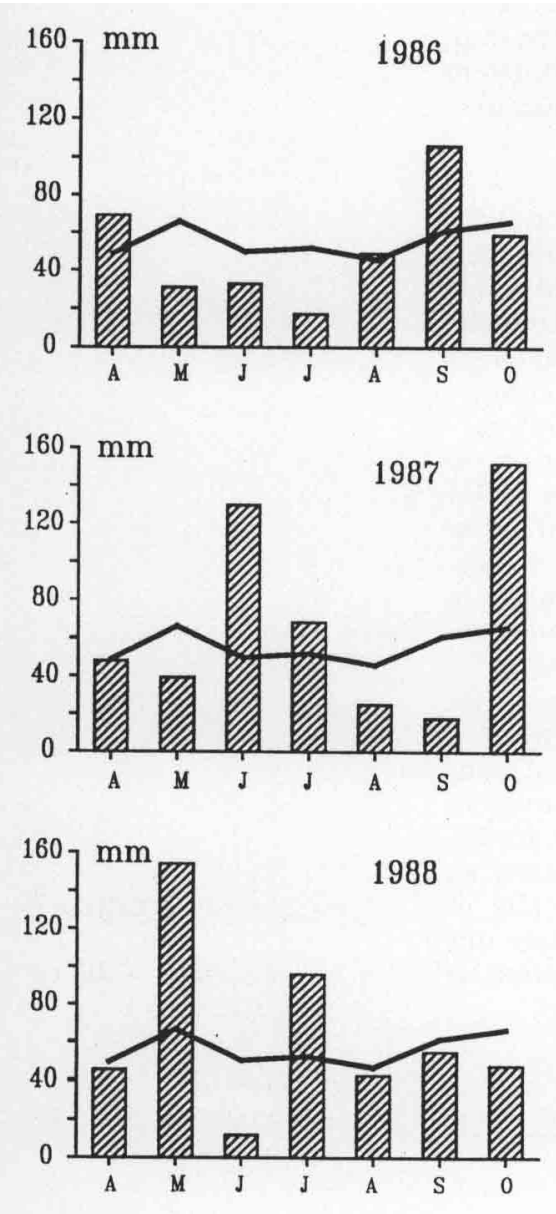
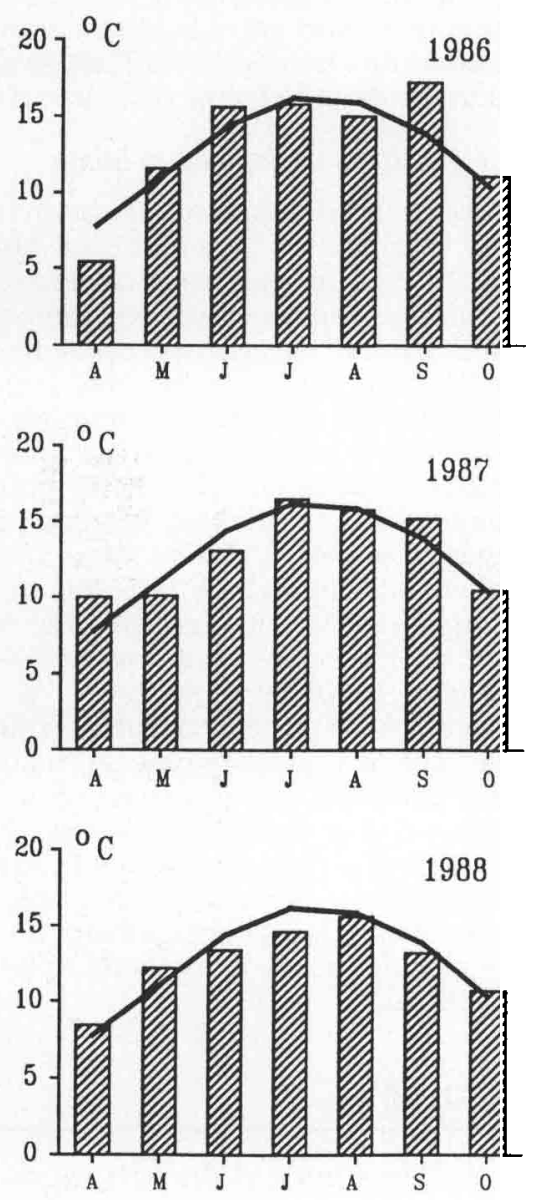

\section{Température}

Figure 2. Calendrier de pâturage du lot Témoin.

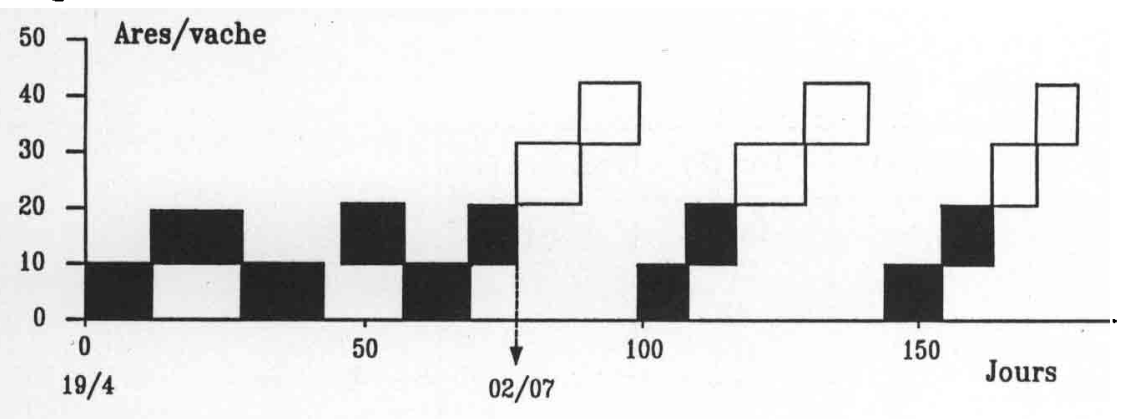

Prairies permanentes

Prairies temporaires

ont généralement été favorables à la production d'herbe sauf durant l'été 1986 où il a alors été nécessaire de distribuer des quantités importantes d'aliments complémentaires (ensilage de maïs) durant environ 8 semaines. Le même phénomène s'est produit durant environ 2 semaines au printemps de 1988 après une période de croissance importante de l'herbe. Ces 2 périodes ont été éliminées des résultats.

Le chargement du lot $\mathrm{T}$ (tableau 1 - figure 2 ) a varié de 20 à 22 ares par vache selon les années au printemps à environ 43 ares en fin de saison. Simultanément les chargements $\mathrm{F}$ et TF sont passés en moyenne de 19 et 16 ares/ vache au printemps à 38 et 33 ares/vache en automne respectivement. En moyenne, pour les 3 années, ceci correspond à 104, 91 et $81 \mathrm{~m}^{2}$ par vache et par jour sur l'ensemble de la saison. Au total 5 cycles de pâturage ont été effectués sur les prairies permanentes (figure 2). La durée de chacun de ceux-ci a augmenté au cours de la saison, le temps de séjour par parcelle variant de 14 jours au printemps (2 parcelles) à 7 jours en automne (4 parcelles). Les refus ont été habituellement fauchés après le $3 \mathrm{e}$ cycle sur les prairies permanentes. Les prairies temporaires ont représenté en moyenne $51 \%$ des surfaces totales (ST) des systèmes mais leur contribution n'a été que d'environ un tiers en terme de jours de pâturage suite à leur récolte de printemps sous forme d'ensilage. Ces surfaces n'ont généralement été fauchées qu'une seule fois (22 mai en moyenne) pour ensuite être pâturées à partir de fin juin. Les quantités de matière sèche (MS) ensilées sur les prairies temporaires ont été d'environ 3,5 t MS/ha soit l'équivalent de $2,4 \mathrm{t}$ MS/ha de surface totale (ST).

\section{2 / Mesures sur l'herbe}

\section{a / Avant pâturage}

Les quantités d'herbe récoltées à chaque entrée sur la parcelle $(n=93)$ en 1987 et 1988 ont été en moyenne de 1,8 t MS/ha (tableau 2). Elles n'ont été influencées $(\mathrm{P}<0,01)$ que par le type de prairies (1,9 t MS en prairies permanentes contre 1,6 en prairies temporaires). La quantité d'herbe allouée a été de 15,6, 13,9 et $11,3 \mathrm{~kg}$ MS respectivement pour les chargements $\mathrm{T}, \mathrm{F}$ et $\mathrm{TF}$.

La moyenne des hauteurs d'herbe à l'entrée des animaux dans chacune des parcelles a été de 11,4 $\pm 3,4 \mathrm{~cm}$ (près de 50000 mesures). Celle-ci n'a pas été différente entre les chargements $T$ et $F$ mais a été inférieure d'environ $1 \mathrm{~cm}(\mathrm{P}<0,05)$ avec le chargement $\mathrm{TF}$ pour les 2 types de prairies.

Comme la biomasse n'était pas différente entre traitements, la quantité d'herbe récoltée par cm de hauteur ou densité $D$ moyenne de l'herbe a été sensiblement plus élevée $(\mathrm{P}<$ 0,10 ) d'environ $6 \%$ avec le chargement TF (respectivement 280,283 et $298 \mathrm{~kg} \mathrm{MS} / \mathrm{cm} / \mathrm{ha}$ pour T, F et TF).

La quantité d'herbe récoltée ( $Y$ en kg MS) est en liaison $(\mathrm{P}<0,01)$ avec la hauteur mesurée à l'entrée (X en $\mathbf{c m})$ mais la relation n'est pas très précise $\left(n=93, R^{2}=0,78\right.$, Syx $\left.=326\right)$. La pente (quantité d'herbe par $\mathrm{cm}$ ) est significativement $(\mathrm{P}<0,01)$ affectée par le chargement. Elle est plus forte avec le chargement TF ce qui est en accord avec la densité de l'herbe calculée précédemment. L'année n'a pas modifié les coefficients de régression mais a influencé uniquement l'ordonnée à l'origine. Les relations Correspondant à chaque chargement sont : 
Tableau 2. Production d'herbe à l'entrée sur les parcelles et hauteurs d'herbe avant et après pâturage (Années 1987 et 1988).

\begin{tabular}{|c|c|c|c|c|c|c|}
\hline \multirow{2}{*}{$\begin{array}{c}\text { Prairies } \\
\text { Chargement }\end{array}$} & \multicolumn{3}{|c|}{$\begin{array}{c}\text { Permanentes } \\
\text { ( } n=20 / \text { traitement })\end{array}$} & \multicolumn{3}{|c|}{$\begin{array}{c}\text { Temporaires } \\
(\mathrm{n}=11 / \text { traitement })\end{array}$} \\
\hline & $\begin{array}{l}\text { Témoin } \\
\text { (T) }\end{array}$ & $\begin{array}{l}\text { Fort } \\
\text { (F) }\end{array}$ & $\begin{array}{l}\text { Très Fort } \\
\text { (TFF] }\end{array}$ & $\begin{array}{l}\text { Témoin } \\
\text { (T) }\end{array}$ & $\begin{array}{l}\text { Fort } \\
(F)\end{array}$ & $\begin{array}{l}\text { Très Fort } \\
\text { (TF] }\end{array}$ \\
\hline A l'entrée des animaux & & & & & & \\
\hline Biomasse (kg MS/ha) (1) & 1872 & 1947 & 1806 & 1597 & 1621 & 1473 \\
\hline Hauteur $(\mathrm{cm})(2)$ & 11,8 & 11,8 & 11,0 & 11,5 & 11,3 & 10,3 \\
\hline A la sortie des animaux & & & & & & \\
\hline Hauteur $(\mathrm{cm})$ & & & & & & \\
\hline - Moyenne (2) & 6,5 & 6,4 & 5,4 & 5,3 & 4,9 & 4,2 \\
\hline - Zones fréquentées & 5,7 & 5,7 & 4,9 & 4,9 & 4,6 & 4,0 \\
\hline - Zones peu fréquentées & 9,6 & 9,5 & 8,8 & 8,4 & 7,8 & 7,1 \\
\hline Importance des zones peu fréquentées (\%) (3) & 20,5 & 18,0 & 14,1 & 12,2 & 11,4 & 6,2 \\
\hline
\end{tabular}

(1) Coupe à la motofaucheuse ( $5 \mathrm{~cm}$ du niveau du sol).

(2) Mesures à l'herbomètre à raison de 150 mesures par hectare et par passage.

(3) Par rapport au nombre total de mesures.

$Y($ Témoin $)=149 \mathrm{X}+36$

$Y$ (Fort) $=203 X-534$

$\mathrm{Y}$ (Très Fort $)=264 \mathrm{X}-1154$

\section{b / Après pâturage}

Les hauteurs moyennes à la sortie ainsi que les proportions de zones de pâturage peu fréquentées ont été plus faibles $(\mathrm{P}<0,01)$ avec le chargement $\mathrm{TF}$ dans les 2 types de prairies (tableau 2). Au contraire, ces paramètres ainsi que les histogrammes de fréquence des hauteurs (figure 3) ont été similaires pour les traitements $\mathrm{T}$ et $\mathrm{F}$. En particulier, les 2 types de zones de fréquentation du pâturage sont comparables et correspondent à des lois de distribution normale (Chi2 toujours inférieur à 0,5 , d.l. = 17). Les mêmes phénomènes ont été observés avec le traitement TF mais à une hauteur inférieure de $1 \mathrm{~cm}$ pour tous les paramètres. Les prairies temporaires se sont caractérisées par une hauteur à la sortie et une proportion de zones peu fréquentées plus faibles qu'avec les prairies permanentes $(-1,3 \mathrm{~cm}$ et 7,6 points $\%$ en moyenne, respectivement).

\section{c / Evolution au cours des cycles}

Durant les 5 cycles de pâturage sur les prairies permanentes, les différents paramètres caractérisant le couvert végétal ont varié de la même manière selon les traitements (tableau 3). Les hauteurs avant le pâturage ont été les plus élevées au printemps, excepté pour le 2 cycle en raison de la faible durée de repos de l'herbe (17 jours). La proportion des zones peu fréquentées a augmenté au cours de la saison (surtout avec les chargements T et F) jusqu'à la fauche des refus après le $3^{\mathrm{e}}$ cycle. L'influence de cette opération sur le profil du couvert végétal a été d'autant plus importante que le chargement était faible. La proportion des zones peu fréquentées a représenté environ $10 \%$ du parcellaire quel que soit le chargement au $4^{\circ}$ cycle de pâturage (tableau 3).

\section{3 / Performances animales}

\section{a / Influence du chargement et du niveau de complémentation}

Les quantités moyennes (162 j) de lait, de matières grasses et de protéines ont été de

Tableau 3. Hauteurs d'herbe et proportion de zones peu fréquentées au cours des 5 cycles de pâturage sur prairies permanentes (1987 et 1988).

\begin{tabular}{|c|c|c|c|c|c|c|c|c|c|c|c|c|c|c|c|c|}
\hline \multirow{2}{*}{\multicolumn{2}{|c|}{$\begin{array}{c}\text { Chargement } \\
\text { ( } \mathrm{n}=31 / \text { traitement) } \\
\text { Numéro de cycle }\end{array}$}} & \multicolumn{5}{|c|}{ Témoin (T) } & \multicolumn{5}{|c|}{ Fort (F) } & \multicolumn{5}{|c|}{ Très Fort (TF) } \\
\hline & & 1 & 2 & 3 & 4 & 5 & 1 & 2 & 3 & 4 & 5 & 1 & 2 & 3 & 4 & $\mathbf{5}$ \\
\hline $\begin{array}{l}\text { Entrée } \\
\text { Animaux }\end{array}$ & Moyenne $(\mathrm{cm})$ & 13,0 & 10,3 & 13,8 & 12,0 & 9,9 & 13,1 & 9,8 & 13,5 & 12,2 & 10,5 & 13,2 & 9,1 & 12,2 & 11,2 & 9,1 \\
\hline \multirow{3}{*}{$\begin{array}{l}\text { Sortie } \\
\text { Animaux }\end{array}$} & Moyenne $(\mathrm{cm})$ & 6,4 & 6,2 & 7,0 & 6,6 & 6,4 & 6,3 & 6,1 & 6,7 & 6,6 & 6,2 & 5,8 & 5,0 & 5,6 & 5,6 & 5,2 \\
\hline & $\begin{array}{l}\text { Zones peu } \\
\text { fréquentées }(\mathrm{cm})\end{array}$ & 10,6 & 10,5 & 9,8 & 9,9 & 8,1 & 10,3 & 10,6 & 10,2 & 9,8 & 7,9 & 9,2 & 9,0 & 9,5 & 9,0 & 7,6 \\
\hline & Importance $(\%)^{[1]}$ & 10,0 & 19,2 & 32,5 & 12,6 & 27,6 & 9,4 & 16,9 & 25,3 & 9,3 & 29,5 & 11,5 & 13,9 & 18,6 & 8,3 & 18,1 \\
\hline
\end{tabular}

(1) par rapport au nombre total de mesures.

Les hauteurs d'herbe à l'entrée et à la sortie des parcelles sont inférieures de $1 \mathrm{~cm}$ environ avec le chargement Très Fort. 
Figure 3. Répartition des hauteurs d'herbe. Répartition des hauteurs d'herbe à l'entrée des parcelles.

Répartition des hauteurs d'herbe en sortie de parcelles selon les zones fréquentées $\square$ ou peu fréquentées $\mathbf{}$.
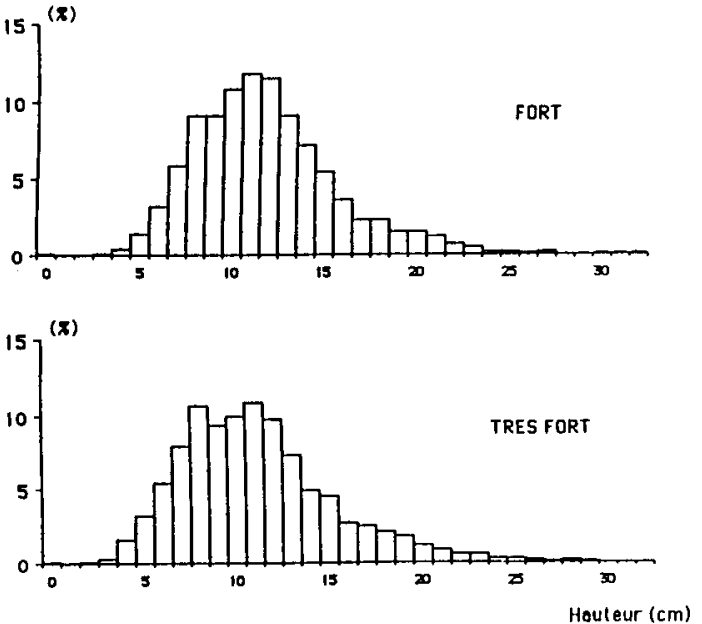

$21,6 \mathrm{~kg}, 814 \mathrm{~g}$ et $656 \mathrm{~g}$ par jour respectivement (tableau 4) pour l'ensemble des traitements. Ces productions n'ont pas été influencées significativement $(\mathrm{P}>0,05)$ par le chargement et n'ont diminué que d'environ $5 \%$ avec le chargement TF. La persistance mensuelle (figure 4) de la production laitière a été plus faible d'environ 1 point par niveau de chargement (88\% pour $\mathrm{T}$ contre $86 \%$ pour $\mathrm{TF}$ ). La composition du lait (taux butyreux et de protéines) n'a pas été affectée par ces traitements. Les variations de poids vif n'ont pas non plus été influencées significativement par le chargement malgré une tendance à une réduction des gains de poids apparents avec l'augmentation du chargement.

Les vaches du niveau $\mathrm{H}$ de complémentation ont consommé en moyenne $3,7 \mathrm{~kg}$ d'aliment
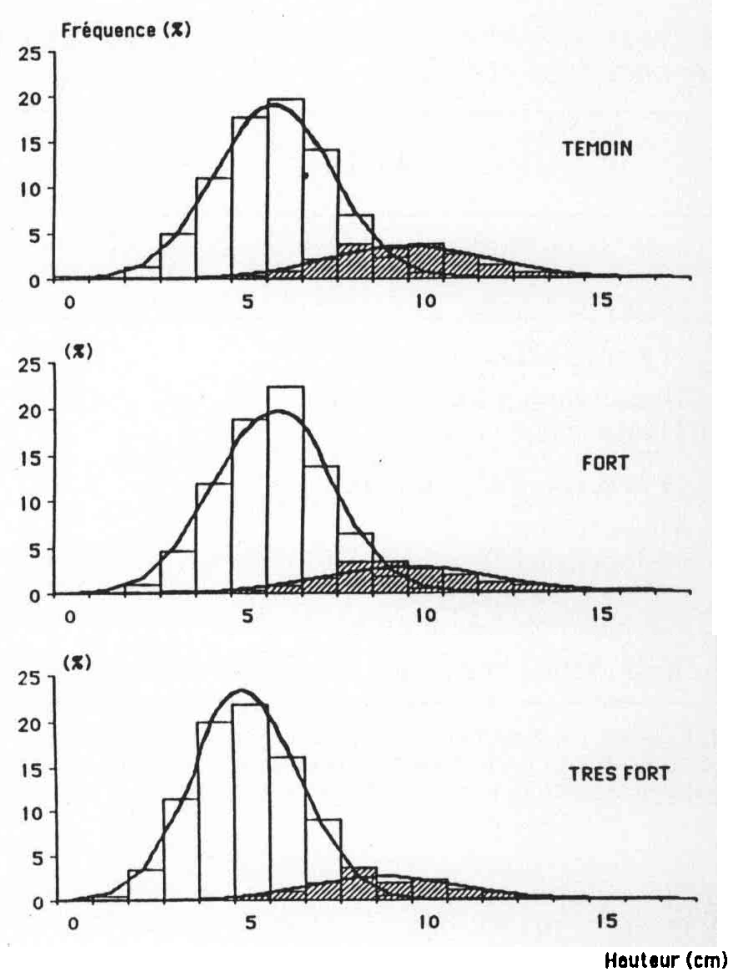

concentré par jour soit $3,2 \mathrm{~kg}$ de plus que celles du niveau $\mathrm{B}$. Les productions individuelles ont toutes été améliorées $(\mathrm{P}<0,01)$ par la complémentation (tableau 4) et son efficacité moyenne a été de $0,6 \mathrm{~kg}$ de lait par $\mathrm{kg}$ d'aliment concentré supplémentaire. Cette efficacité a été sensiblement plus élevée avec le chargement TF (+ $0,82 \mathrm{~kg}$ lait $/ \mathrm{kg}$ d'aliment contre $+.0,50$ pour les traitements $\mathrm{T}$ et $\mathrm{F}$ ) en particulier chez les vaches les plus fortes productrices $(\mathrm{P}<0,10)$. Les persistances mensuelles de la production laitière ont été de 89 et $85 \%$ respectivement pour les niveaux $\mathrm{H}$ et $\mathrm{B}$ de complémentation (figure 4).

La teneur en protéines du lait $(+0,6 \mathrm{~g} / \mathrm{kg})$ a été significativement améliorée $(\mathrm{P}<0,01)$ par la complémentation, mais le taux butyreux n'a

Tableau 4. Performances individuelles journalières (Moyennes ajustées -54 vaches/traitement $\times 162$ jours).

\begin{tabular}{|c|c|c|c|c|c|c|}
\hline & \multicolumn{3}{|c|}{ Chargement } & \multicolumn{2}{|c|}{ Concentré } & \multirow[b]{2}{*}{ ETR } \\
\hline & $\begin{array}{l}\text { Témoin } \\
\text { (T) }\end{array}$ & $\begin{array}{c}\text { Fort } \\
\text { (F) }\end{array}$ & $\begin{array}{l}\text { Très Fort } \\
\text { (TF) }\end{array}$ & $\underset{(\mathrm{H})}{\text { Haut }}$ & $\begin{array}{l}\text { Bas } \\
(\mathbf{B})\end{array}$ & \\
\hline $\begin{array}{l}\text { Lait }(\mathrm{kg}) \\
\text { Lait } 4 \%(\mathrm{~kg})\end{array}$ & $\begin{array}{l}22,1 \mathrm{a} \\
21,3 \mathrm{a}\end{array}$ & $\begin{array}{l}21,7 \mathrm{a} \\
21,0 \mathrm{a}\end{array}$ & $\begin{array}{l}21,1 \mathrm{a} \\
20,3 \mathrm{a}\end{array}$ & $\begin{array}{l}22,6 \mathrm{~A} \\
21,8 \mathrm{~A}\end{array}$ & $\begin{array}{l}20,7 \mathrm{~B} \\
20,0 \mathrm{~B}\end{array}$ & $\begin{array}{l}2,17 \\
2,07\end{array}$ \\
\hline $\begin{array}{l}\text { Matières grasses (g) } \\
\text { Matières protéiques (g) } \\
\text { Taux butyreux (g \%o) } \\
\text { Taux protéique (g \%o) }\end{array}$ & $\begin{array}{l}832 \mathrm{a} \\
662 \mathrm{a} \\
37,7 \mathrm{a} \\
30,5 \mathrm{a}\end{array}$ & $\begin{array}{l}821 \mathrm{a} \\
663 \mathrm{a} \\
37,8 \mathrm{a} \\
30,6 \mathrm{a}\end{array}$ & $\begin{array}{r}790 \mathrm{a} \\
642 \mathrm{a} \\
37,6 \mathrm{a} \\
30,6 \mathrm{a}\end{array}$ & $\begin{array}{l}848 \mathrm{~A} \\
691 \mathrm{~A} \\
37,6 \mathrm{~A} \\
30,9 \mathrm{~A}\end{array}$ & $\begin{aligned} 781 \mathrm{~B} \\
620 \mathrm{~B} \\
37,8 \mathrm{~A} \\
30,3 \mathrm{~B}\end{aligned}$ & $\begin{array}{l}87,9 \\
68,0 \\
2,38 \\
1,44\end{array}$ \\
\hline $\begin{array}{l}\text { Poids vif }(\mathrm{kg}) \\
\text { Variation de poids vif }(\mathrm{g} / \mathrm{j})\end{array}$ & $\begin{array}{l}635 \mathrm{a} \\
83 \mathrm{a}\end{array}$ & $\begin{array}{c}631 \mathrm{a} \\
47 \mathrm{a}\end{array}$ & $\begin{array}{l}630 \mathrm{a} \\
24 \mathrm{a}\end{array}$ & $\begin{array}{l}636 \mathrm{~A} \\
80 \mathrm{a}\end{array}$ & $\begin{array}{l}628 \mathrm{~B} \\
23 \mathrm{~b}\end{array}$ & $\begin{array}{c}20,1 \\
153,7\end{array}$ \\
\hline
\end{tabular}

Pour chaque variable, l'analyse statistique a été réalisée en intégrant dans le modèle les interactions de pente significative. Les moyennes suivies de lettres différentes sont significativement différentes (minuscules : P $<0,05 ;$ majuscules : $\mathrm{P}<0,01\}$. 
pas été affecté $\{P>0,05\}$. Cette amélioration du taux protéique a été d'autant plus sensible que le chargement été élevé (jusqu'à $+0,9$ $\mathrm{g} / \mathrm{kg}$ ). La complémentation a amélioré le gain de poids vif moyen d'environ $10 \mathrm{~kg}(\mathrm{P}<0,01)$ sur l'ensemble de la saison.

Ainsi que le montre la figure 5, la complémentation a été d'autant plus efficace que le niveau de production était élevé (interaction significative $\mathrm{P}<0,01)$. La réponse marginale a été de $0,26 \mathrm{~kg}$ de lait par $\mathrm{kg}$ de lait supplémentaire produit à la mise à l'herbe. Ainsi, l'effet de la complémentation a été pratiquement nul jusqu'à $22 \mathrm{~kg}$ de lait, mais a atteint plus de $3 \mathrm{~kg}$ pour des vaches produisant $35 \mathrm{~kg}$ de lait à la mise à l'herbe.

\section{b / Variations journalières des productions durant le temps de séjour sur les parcelles}

Les productions de lait, de matières grasses et de protéines ont évolué de façon cyclique durant le temps de séjour sur chacune des parcelles (figures 2 et 6 ). Ces variations ont été

Figure 4. Evolution journalière de la production laitière.

a) selon le niveau de chargement

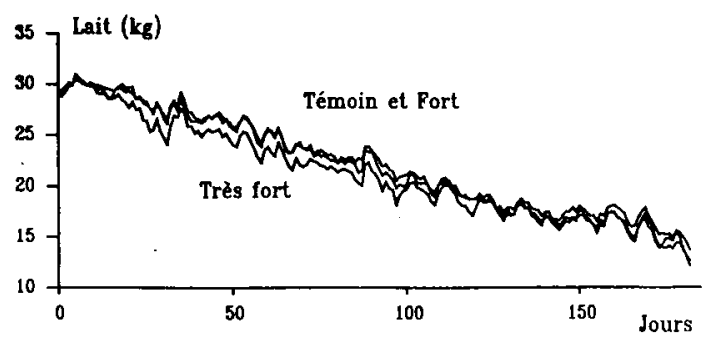

b) selon le niveau de complémentation

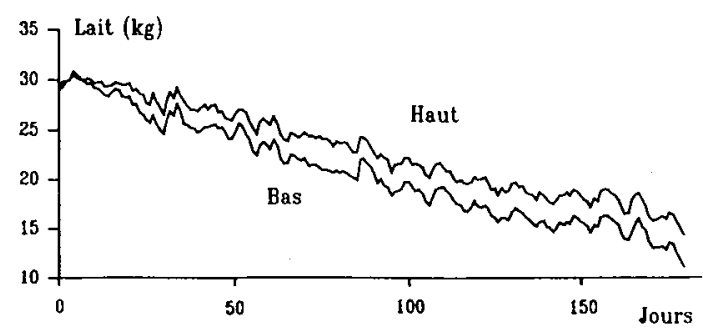

Figure 5. Relation entre la quantité de lait produite pendant l'essai et le niveau de production en préexpérience selon l'apport ou non d'aliment concentré.

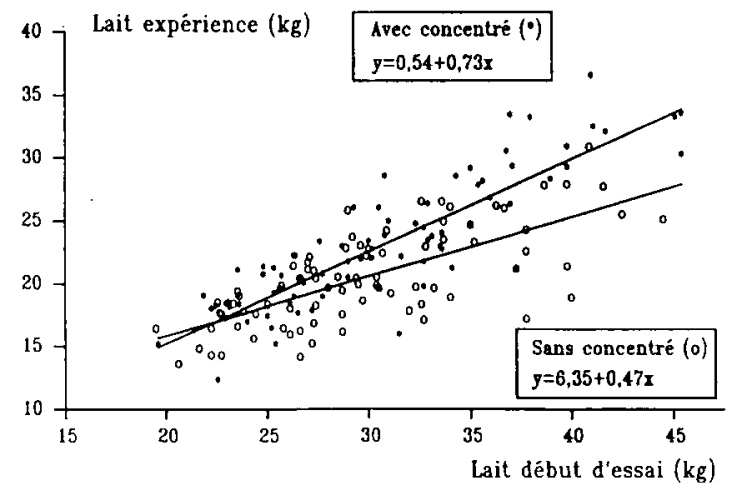

Figure 6. Variations des productions de lait durant le temps de séjour sur une parcelle. a) Selon le niveau de chargement

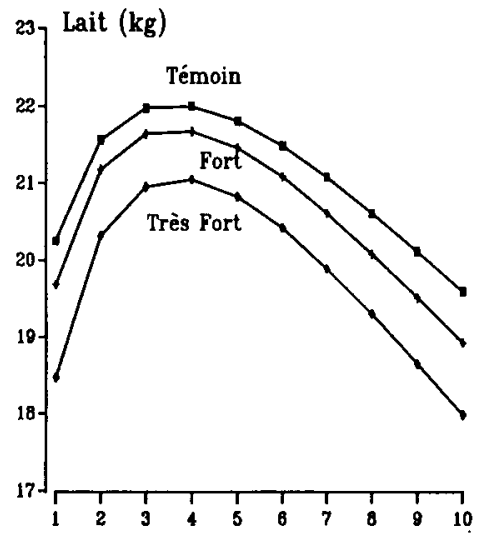

$\begin{array}{ll}\text { - Témoin } & y=21.1 \times \mathrm{n}^{0.151} \times \mathrm{e}^{-0.042 \mathrm{n}} \\ \text { + Fort } & \mathrm{y}=20.7 \times \mathrm{n}^{0.177} \times \mathrm{e}^{-0.050 \mathrm{n}} \\ \text { - Très Fort } & y=19.6 \times \mathrm{n}^{0.225} \times \mathrm{e}^{-0.081 \mathrm{n}}\end{array}$ b) Selon le niveau de complëmentation

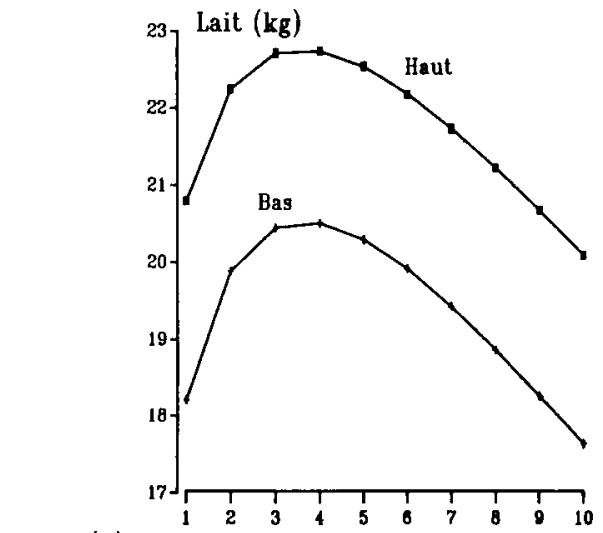

Jours (n)

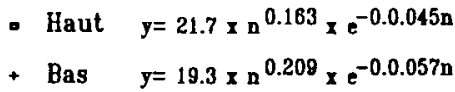

+ Bas $y=19.3 \times \mathrm{n}^{0.209} \times \mathrm{e}^{-0.0 .057 \mathrm{n}}$ analysées selon le modèle proposé par Wood (1976) pour la courbe de la lactation : $Y_{n}=A x$ $\mathrm{n}^{\mathrm{B}} \times \mathrm{e}^{-\mathrm{ch}}$ (Hoden et al $1986 \mathrm{~b}, \mathrm{c}$ ). Les données journalières de productions des 31 temps de séjour au cours des 3 années ont été ramenées à la durée moyenne de 10 jours. Les valeurs maximales des quantités de lait produites ont varié de 105 à $108 \%$ de la valeur moyenne et ont été observées entre les jours 3 et 4 . En raison même des règles de conduite du pâturage, le rapport des productions entre les 2 derniers et les 2 jours maximum $(\mathrm{min} / \mathrm{max})$ a été de 0,90 pour le traitement T. Ce rapport n'a pas été très différent pour les autres chargements $\{0,89$ et 0,87 respectivement pour $F$ et $\mathrm{TF}$ ) qui n'ont pas été conduits selon leurs propres variations de la production laitière. Les augmentations en début de parcelle et les diminutions durant le temps de séjour ont cependant eu tendance à être plus importantes avec les chargements les plus élevés. Par ailleurs, le rapport min/max n'a pas été affecté par le niveau de complémentation qui n'a pas eu un effet tampon important sur la production laitière durant le temps de séjour.

Les variations des quantités de protéines synthétisées ont été plus importantes. Tous chargements confondus, le rapport $\min / \max$ a atteint 0,85 contre 0,89 pour le lait et les matières grasses. En particulier, le taux protéique a été plus faible de $1,4 \mathrm{~g} / \mathrm{kg}$ en fin de séjour comparativement à celui observé au maximum lors des jours 3 et 4 .

\section{c / Estimation des quantités d'herbe ingérées}

Les principaux résultats obtenus uniquement en chargement $\mathrm{T}$ et TF sont présentés au tableau 5. La digestibilité (DMO) de l'herbe n'a pas été affectée $(\mathrm{P}>0,05)$ par les traitements mais a été significativement réduite $(\mathrm{P}<0,05)$ durant le séjour $(0,80$ au milieu contre 0,75 à la fin). 
Estimation des quantités ingérées en chargements Témoin et Très Fort (Moyennes de 7 à 9 vaches/traitement au cours de 4 jours par période)

\begin{tabular}{|c|c|c|c|c|c|c|c|c|}
\hline & \multicolumn{4}{|c|}{ Chargement } & \multirow[b]{3}{*}{ ETR } & \multirow{2}{*}{\multicolumn{3}{|c|}{$\begin{array}{l}\text { Temps de séjour } \\
\text { dans la parcelle }\end{array}$}} \\
\hline & \multirow{2}{*}{\multicolumn{2}{|c|}{ 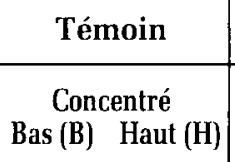 }} & \multirow{2}{*}{\multicolumn{2}{|c|}{$\begin{array}{c}\text { Très Fort } \\
\begin{array}{c}\text { Concentré } \\
\text { Bas (B) Haut }(\mathrm{H})\end{array}\end{array}$}} & & & & \\
\hline & & & & & & Milieu & Fin & ETR \\
\hline $\begin{array}{l}\text { Différences de concentrés } \\
\text { ingérées (kg } \mathrm{MO} / \mathrm{vache} / \text { jour) }\end{array}$ & \multicolumn{2}{|c|}{2,2} & \multicolumn{2}{|c|}{2,3} & & & 0,0 & \\
\hline Hauteur d'herbe $(\mathrm{cm})$ & \multicolumn{2}{|c|}{8,7} & \multicolumn{2}{|c|}{7,8} & 0,23 & 9,9 & 6,7 & 1,09 \\
\hline $\begin{array}{l}\text { Digestibilité de la MO } \\
\text { de l'herbe pâturée (1) }\end{array}$ & \multicolumn{2}{|c|}{0,78} & \multicolumn{2}{|c|}{0,77} & 0,082 & 0,80 & 0,75 & 0,196 \\
\hline $\begin{array}{l}\text { Quantités totales ingérées } \\
\text { (kg MO/vache/jour) }\end{array}$ & 17,6 & 18,4 & 15,7 & 18,3 & 0,87 & 18,5 & 16,5 & 2,11 \\
\hline $\begin{array}{l}\text { Quantités d'herbe ingérées } \\
\text { (kg MO/vache/jour) }\end{array}$ & 17,6 & 16,3 & 15,7 & 15,9 & 0,89 & 17,4 & 15,4 & 2,35 \\
\hline Lait (kg/vache/jour) & 23,2 & 25,4 & 21,6 & 24,6 & 1,16 & 26,0 & 21,4 & 3,47 \\
\hline
\end{tabular}

(1) Estimation à partir des vaches du niveau B de concentré (cf texte).

Les quantités d'herbe ingérées ( $\mathrm{kg} \mathrm{MO}$ ) et la production laitière ont diminué simultanément durant les mêmes périodes avec le chargement $\mathrm{TF}$, en particulier chez les vaches du niveau $\mathrm{B}$ de complémentation $(\mathrm{P}<0,05)$. Une interaction significative $(P<0,05)$ a été mise en évidence entre le chargement et le niveau de concentré sur les quantités d'herbe et la ration totale ingérées. L'ingestion d'herbe a été réduite de $1,3 \mathrm{~kg}$ chez les vaches du chargement $\mathrm{T}$ recevant de l'aliment concentré (taux de substitution de 0,6) tandis qu'elle n'a pas été modifiée avec le chargement TF. En conséquence, l'addition d'aliment concentré a permis une augmentation de la ration totale de seulement $0,8 \mathrm{~kg}$ avec le chargement $\mathrm{T}$ et de $2,6 \mathrm{~kg}$ avec le chargement TF. Pour les 2 niveaux de complémentation, l'ingestion totale a diminué d'environ $2 \mathrm{~kg}$ à la fin du temps de séjour, mais la différence n'est pas significative $(P<0,10)$ en raison d'une grande variabilité entre périodes.

\section{d / Production globale par hectare}

Les quantités de lait $4 \%$ produites sur l'ensemble du système durant toute la saison ont augmenté avec le chargement (tableau 6). En plus des 2,4 t MS récoltées /ha, les productions globales ont été d'environ 8000,9000 et 9800 $\mathrm{kg} / \mathrm{ha}$ pour les traitements $\mathrm{T}$, F et TF soit des valeurs relatives de 100,112 et $123 \%$. Les accroissements de matières protéiques et de matière utile (matières grasses + protéines) synthétisées ont été du même ordre de grandeur. Il en a été sensiblement de même en termes de lait « autonome » et d'énergie " productible » qui prennent en compte les aliments complémentaires distribués et les récoltes des excédents de printemps.

\section{Discussion et conclusion}

\section{Effets du chargement}

L'objectif principal de cet essai pluriannuel était d'examiner l'effet du chargement sur les performances de production. L'échelle de variation de ce facteur avait volontairement été choisie dans une zone permettant de ne pas trop pénaliser les performances individuelles. Ainsi le chargement témoin correspondait à celui habituellement pratiqué $(4,7$ vaches au printemps et 2,3 en automne) dans les conditions de la station du Pin au Haras (Hoden et al 1986 b, c ; Hoden et Muller 1989).

Tableau 6. Bilan global de pâturage sur la surface totale (Moyennes arithmétiques par hectare sur 162 jours par année).

\begin{tabular}{|lccc|}
\hline \multicolumn{1}{|c}{ Chargement } & Témoin (T) & Fort (F) & Très Fort (TF) \\
\hline Lait 4 \% produit (kg) & 7970 & 8961 & 9816 \\
Ensilage récolté (kg MS) & 2400 & 2400 & 2400 \\
Aliment concentré (kg MS) & 636 & 683 & 773 \\
Ensilage de maïs (kg MS) (1) & 342 & 397 & 466 \\
Lait 4 \% autonome (kg) & 5734 & 6500 & 6997 \\
Energie productible (UFL) & 5954 & 6555 & 7057 \\
\hline
\end{tabular}

(1) durant la période de transition à la mise à I'herbe. 
Tableau 5. Approche de la valorisation du pâturage sur la surface totale du système (à partir des données 1987 et 1988).

\begin{tabular}{|lccc|}
\hline \multicolumn{1}{|c}{ Chargement } & Témoin (T) & Fort (F) & Très Fort (TF) \\
\hline Surface offerte - S (m²/vache/jour) (1) & 100 & 88 & 77 \\
Hauteur d'herbe disparue - HD (cm) (2) & 5,6 & 5,8 & 5,8 \\
Densité de l'herbe - D (kg MS/cm/ha) (3) & 280 & 283 & 298 \\
Index d'herbe ingérée - S × HD × D & $15,7(100)$ & $14,4(92)$ & $13,3(85)$ \\
\hline
\end{tabular}

(1) = Surface totale du système / nombre de jours de pâturage

(2) = Hauteur (Entrée-Sortie) mesurée à l'herbomètre.

(3) = Biomasse Entrée (Tableau 2) /(Hauteur Entrée $-5 \mathrm{~cm}$ ).

\section{Approche de la valorisation du couvert végétal selon les chargements}

Les chargements se traduisent par des différences de biomasse offerte conventionnellement définies comme étant les quantités d'herbe allouées par vache et par jour à l'entrée sur chaque parcelle. Leurs valeurs relatives ont été de 100, 89 et $72 \%$ respectivement pour les traitements $T, F$ et TF alors que la réduction des performances individuelles n'a été que de $5 \%$ entre les chargements extrêmes. Ce critère ainsi calculé ne représente pas la biomasse réelle valorisable par les animaux. Wade et al (1989 et 1991) ont montré que la quantité d'herbe réellement ingérée était en relation avec $1 /$ le volume pâturé qui est le produit de la surface offerte par la profondeur de pâturage et 2/ la densité brute moyenne de la strate pâturée. Une telle approche peut être effectuée grâce aux mesures systématique réalisées sur ces paramètres (tableau 7). La surface offerte $S$ correspond au chargement. La profondeur de pâturage ou la hauteur d'herbe disparue (HD) peut être estimée par différence entre les mesures d'entrée et de sortie du pâturage réalisées à l'herbomètre. Entre traitements, HD est peu différente $(5,5$ à $5,7 \mathrm{~cm})$ bien que la hauteur de l'herbe soit plus faible de $1 \mathrm{~cm}$ avec le chargement TF. Enfin la densité $D$ de la strate pâturée augmente en moyenne de $6 \%$ entre les chargements T et TF. Il est ainsi possible de calculer un "index d'herbe ingérée " (en kg MS/ vache/j) qui est le produit de $S \times H D \times D$. Les variations relatives de la production laitière selon le chargement semblent déjà mieux expliquées par cet index correspondant à 100, 92 et $85 \%$ respectivement pour les traitements $T, F$ et TF. Ce critère synthétique sous-estime cependant l'ingestion réelle d'herbe car il ne prend en compte ni la croissance de l'herbe durant le temps de séjour ni les éventuels fourrages complémentaires distribués. De plus, il est aussi possible que des vaches pâturent en dessous de la hauteur de coupe de la motofaucheuse (c'est à dire $5 \mathrm{~cm}$ ). Néanmoins, cet index et les variations relatives de ses composants montrent les possibilités d'adaptations simultanées de la vache et du couvert végétal à l'augmentation du chargement, du moins dans une gamme de variation raisonnable (Holmes 1989 b).

Par ailleurs, pour expliquer les variations entre chargements, il serait aussi nécessaire de tenir compte de la digestibilité de l'herbe sélectionnée. Les résultats issus de la technique des index fécaux ont montré que la digestibilité de l'herbe n'avait pas été affectée dans le chargement TF bien que la hauteur après pâturage soit inférieure de $1 \mathrm{~cm}$ par rapport au chargement $T$. Ceci indiquerait que le couvert végétal s'est adapté au traitement TF aussi bien en termes de digestibilité que de densité. Ces hypothèses seraient en accord avec les résultats de Jackson (1976) qui a montré qu'à niveau constant de fauche, la hauteur moyenne d'insertion de la feuille verte la plus basse diminue avec celle de la coupe.
En système de pâturage tournant simplifié, l'élévation du chargement de $29 \%$ (3,0 vs 2,3 vaches par ha de surface totale) s'est traduite par une réduction ( $5 \%$ ) non significative des performances individuelles mais un accroissement des productions par ha d'environ $23 \%$. Ces résultats correspondent à ceux de la bibliographie (Journet et Demarquilly 1979; Mayne et al 1987, 1988 ; Holmes 1989 a, b) aux alentours du chargement dit "optimum " et montrent que le niveau TF n'était pas trop sévère. Cette conclusion est en accord avec les données de Mayne et al (1987) obtenues en pâturage tournant. Ces auteurs indiquent en effet qu'une hauteur d'herbe à la sortie du pâturage située entre 5 et $6 \mathrm{~cm}$ n'est pas un facteur limitant de la production laitière et permet d'obtenir un accroissement des performances globales avec l'augmentation du chargement. Ces résultats montrent aussi que les données obtenues en système de pâturage rationné ou continu peuvent être généralisées au pâturage tournant simplifié. Ils présentent l'avantage d'avoir été obtenus à partir des mêmes prairies durant 3 saisons consécutives avec des effectifs importants de vaches à haut niveau de production.

\section{Effets de la complémentation}

Les réponses moyennes supplémentaires de production laitière (environ $0,6 \mathrm{~kg}$ lait $/ \mathrm{kg}$ concentré) et de gain de poids vif $(+60 \mathrm{~g} / \mathrm{j}$ sur la saison) liées à la complémentation ainsi que la tendance à l'amélioration de son efficacité avec l'accroissement du chargement sont en accord avec les données obtenues par ailleurs
L'efficacité du concentré est en moyenne de $0,6 \mathrm{~kg}$ de lait par $\mathrm{kg}$ d'aliment et s'améliore avec le chargement et le niveau de production des vaches. 
(revues de Journet et Demarquilly 1979, Mayne et Gordon 1984, Leaver 1985). Cependant, l'interaction entre l'apport d'aliment concentré et la quantité de lait produite en préexpérience montre clairement que les réponses moyennes dépendent du potentiel de la vache. En fait, l'efficacité de la complémentation s'est avérée pratiquement nulle chez les vaches dont le niveau de production était inférieur à $25 \mathrm{~kg}$ de lait à la mise à l'herbe. Par rapport aux quantités d'aliment concentré réellement ingérées (+ $290 \mathrm{~g} / \mathrm{kg}$ lait au dessus de $20 \mathrm{~kg}$ de lait lors de la mise à l'herbe), l'efficacité de la complémentation (kg lait/kg supplémentaire de concentré) est passée de 0,50 à 0,70 et 0,75 pour des vaches produisant à la mise à l'herbe 25,30 et $35 \mathrm{~kg}$ de lait respectivement. Ces données sont en accord avec les propositions pratiques faites par ailleurs (Hoden et al 1987; INRA 1988) concernant les niveaux de complémentation et les possibilités de simplification de l'apport d'aliment concentré au pâturage.

\section{Conduite du pâturage tournant simplifié}

Les variations journalières moyennes des productions de lait durant le temps de séjour sur une parcelle donnée ont été comparables à celles observées précédemment (Hoden et al $1986 \mathrm{~b}$ et c) et dans d'autres conditions (Garel et Hoden 1985 ; Hoden et al 1987). Elles traduisent bien la sensible diminution des quantités d'énergie ingérées en fin du temps de séjour. Les 5 périodes de mesure effectuées montrent que les estimations de quantités de matière organique totale ingérées ont été en moyenne de $17,5 \mathrm{~kg}$ pour une production laitière de 23,7 $\mathrm{kg}$. Ces estimations de l'ingestion sont corrélées $\left(\mathrm{R}^{2}=0,49 ; \mathrm{n}=20\right)$ avec la production de lait durant les mêmes périodes et sont en accord avec celles mentionnées par d'autres auteurs (Kibon et Holmes 1987; Comeron 1991 ; Wade 1991). Ces estimations indiquent aussi que la valeur alimentaire de l'herbe (quantités ingérées $x$ digestibilité) diminue entre le milieu et la fin du séjour d'environ $2 \mathrm{~kg}$ de matière organique soit 2,5 UFL/jour. Les apports sont globalement excédentaires de 2 à 3 UFL par rapport aux besoins des animaux (INRA, 1988). Ceci peut être dû à l'effet du pâturage qui accroît de 10 à $50 \%$ le besoin d'entretien, à la non-prise en compte du gain de poids vif des animaux et à une légère surestimation liée à la méthode utilisée. De fait, il est préférable de s'attacher plus aux différences relatives entre les traitements en gardant à l'esprit que 5 périodes de mesures de 4 jours ne peuvent être représentatives de 3 saisons de pâturage consécutives.

Le changement de parcelle, basé sur la diminution de $10 \%$ de la production laitière dans le traitement $\mathrm{T}$, a correspondu à une hauteur d'herbe après pâturage peu variable (en moyenne $6,5 \pm 0,4 \mathrm{~cm}$ en prairies permanentes). Mayne et al (1987, 1988) avaient proposé d'utiliser ce critère en tant que facteur de décision de changement de parcelle. Il semble que les 2 approches soient en bon accord et également applicables. La production est aisée à estimer globalement au niveau du tank à lait. L'utilisation de la hauteur d'herbe résiduelle nécessite de tenir compte du type de prairie. Elle a été de $1 \mathrm{~cm}$ inférieure dans le cas des prairies temporaires par rapport aux permanentes. De plus, la grande variabilité de l'estimation de la mesure (environ $35 \%$ ) nécessite d'effectuer au moins 50 mesures par hectare pour obtenir une précision de l'estimation de $1 \mathrm{~cm}$.

Les variations journalières importantes des productions et de la composition du lait, liées au faible nombre de parcelles, ne sont pas sans poser de difficultés quand les mesures sont effectuées périodiquement au sein des troupeaux (contrôle laitier, laiterie...). Afin de réduire cette variabilité journalière et avoir plus de souplesse dans la gestion du pâturage, il est envisagé d'utiliser un dispositif à 3 parcelles au printemps et passant à 5 ou 6 parcelles en fin de saison. Cette technique permet néanmoins de conserver les avantages de la simplification du système de conduite.

En conclusion, il est possible d'ajuster le chargement à un niveau élevé dans un juste compromis pour augmenter la production de lait par hectare sans pénaliser les performances individuelles des vaches fortes productrices. L'efficacité de la complémentation, d'environ $0,6 \mathrm{~kg}$ de lait par $\mathrm{kg}$ d'aliment concentré, tend à s'améliorer avec le niveau de production et l'augmentation du chargement. La réalisation de façon systématique de mesures simples telles que la hauteur de l'herbe et la biomasse présente ont permis d'avoir une meilleure approche de l'utilisation de l'herbe par les vaches.

\section{Remerciements}

Les auteurs tiennent à remercier leur collègue M.H. Wade, chercheur anglais à la Station de Recherches sur la Vache Laitière, pour ses conseils avisés concernant l'approche de la valorisation du couvert végétal par les animaux. Cet article a été publié en anglais dans la revue Journal of Agricultural Science, Cambridge, 1991 - Vol. 116, p. 417-428. 


\section{Références bibliographiques}

CASTLE M.E., 1976. A simple disc instrument for estimating herbage yield. J. Br. Grassld Soc., 31, 37-40. COMERON E.A., 1991. Estimation des quantités ingérées par les vaches laitières au pâturage. Influence des caractéristiques des animaux, de la quantité et de la structure de l'herbe offerte. Thèse de Doctorat de l'Université de Rennes I. N d'ordre 572.

GAREL J.P., HODEN A., 1985. Pâturage en zone de montagne: niveaux de chargement et de complémentation pour des vaches laitières. Bull. Tech. C.R.Z.V. Theix, INRA, 62, 35-46.

HODEN A., MICOL D., LIENARD G., MULLER A., PEYRAUD J.L., 1986a. Interprétation des essais de pâturage avec des bovins: terminologie, modes de calcul, bilans annuels. Bull. Tech. C.R.Z.V. Theix, INRA, 63, 31-42. HODEN A., MULLER A., IOURNET M., FAVERDIN P., 1986b. Pâturage pour vaches laitières. 1. Comparaison des systèmes de pâturage rationné et tournant simplifié en zone normande. Bull. Tech. C.R.Z.V. Theix, INRA, 64, 25-35

HODEN A., MULLER A., JOURNET M., FAVERDIN P., 1986c. Pâturage pour vaches laitières. 2. Simplification des systèmes de pâturage et niveaux de complémentation en zone normande. Bull. Tech. C.R.Z.V. Theix, INRA, 66, 5-16.

HODEN A FIORELLI JI, HUGUFT L, MULLER A WEISS P., JEANNIN B., 1987. Le pâturage simplifié pour vaches laitières: synthèse des résultats expérimentaux. Bull. Tech. C.R.Z.V. Theix, INRA, 69, 55-65 et Fourrages $111,239-257$

HODEN A., MULLER A., 1989. Simplification des systèmes de pâturage pour vaches laitières. XVIe Congrés International des Herbages, Nice, 1149-1150.

HOLMES W., 1989a. Application on the farm. In Grass, its production and utilization. Ed W. Holmes (2nd ed.), Blackwell, Oxford. 258-271.

HOLMES W., 1989b. The utilization of pasture. In Ruminant Nutrition: Recommended allowances and feed tables. Ed R.Jarrige, 181-192. INRA, John Libbey, Londres \& Paris.

INRA, 1988. Alimentation des bovins, ovins et caprins. Ed R.Jarrige. INRA Publications, 78000 Versailles.

JACKSON D.K., 1976. The influence of patterns of defoliation on sward morphology. In Pasture utilisation by the grazing animal. Ed J.Hodgson \& D.K. Jackson, 51-60. British Grassland Society Occasional Symposium N ${ }^{\circ} 8$.
JOURNET M., DEMARQUILLY C., 1979. Grazing. In Feeding strategy for the high yielding cow. Ed W.H. Broster \& H. Snan, Canada Publishing Co, St Albans, 295-321.

KIBON A., HOLMES W., 1987. The effect of heigh pasture and concentrate composition on dairy cows grazed on continuously stocked pastures. J. Agric. Sci., Cambridge, 109, 293-301.

LEAVER J.D., 1985. Review - article - Milk production from grazed temperate grassland. J. Dairy Res., 52, 313344

MAYNE C.S., GORDON F.J., 1984. The effect of the type of concentrate and level of concentrate feeding on milk production. Anim. Prod., 39, 65-76.

MAYNE C.S., NEWBERRY R.D., WOODCOCK S.C.F., WILKINS R.J,, 1987. Effect of grazing severity on grass utilisation and milk production of rotationally grazed dairy cows. Grass and Forage Sci., 42, 59-72.

MAYNE C.S., NEWBERRY R.D., WOODCOCK S.C.F, 1988. The effect of a flexible grazing management strategy and leader/follower grazing on the milk production of grazing dairy cows and on sward characteristics. Grass and Forage Sci., 43, 137-150.

MOTT G.O., 1960. Grazing pressure and the measurement of pasture production. Proceedings of the 8th International Grassland Congress, Reading, 606-611.

PEYRAUD JL, COMFRON E A WADE MH 1989. Some factors affecting herbage intake of high yielding dairy cows at grazing. XVI ${ }^{\circ}$ Congrès International des Herbages, Nice, 1151-1152.

SAS Institute, Inc., 1987. SAS/STAT Guide for Personnal Computer Version 6 Edition ,Cary N.C.

WADE M.H., 1991. Factors affecting the availability of vegetative Lolium perenne to grazing dairy cows with special reference to sward characteristics, stocking rate and grazing method. Thèse de Doctorat de l'Université de Rennes I. N ${ }^{\cup}$ d'ordre 615.

WADE M.H., PEYRAUD J.L., LEMAIRE G., COMERON E.A., 1989. The dynamics of daily area and depth of grazing, herbage intake of cows in a five day paddock system. XVI Congrès International des herbages, Nice, 1111-1112.

WOOD P.D.P., 1976. Algebraic models of the lactation curves for milk, fat and protein production with estimates of seasonal variation. Anim. Prod., 22, 35-40.

\section{Summary}

Simplified rotational grazing management of dairy cows : Effects of rates of stocking and concentrate

An experiment was carried out in Normandy between 1986 and 1988 over c. 6 months in each year, using a simplified rotational grazing technique (two paddocks in spring, four in autumn) with a total of 162 high-yielding cows (mean milk yield of $30.0 \mathrm{~kg} / \mathrm{day}$ at turn-out). Three stocking rates, designated control ( $T$ : $2.3 \mathrm{cows} / \mathrm{ha}$ over the total area of the system), moderate ( $F: 2.6$ cows $/$ ha $=115 \%$ of $T$ ) and high (TF : 3.0 cows $/$ ha $=130 \%$ of $\mathrm{T}$ ), were compared at two rates of concentrate supplement, $3.7 \mathrm{~kg} / \mathrm{cow}$ per day $(\mathrm{H})$ and $0.5 \mathrm{~kg} / \mathrm{cow}$ per day (B) . Individual milk yield differed by only a non significant $5 \%$ between the extreme stocking rates $(21.3 \mathrm{v} .20 .3 \mathrm{~kg}$ fat-corrected milk (FCM) for treatments $\mathrm{T}$ and $\mathrm{TF}$, respectively). Milk production/ha was $23 \%$ greater in the TF treatment $(9816 \mathrm{v} .7970 \mathrm{~kg}$ FCM for TF and T, respectively). Concentrate supplementation led, on average, to a $9 \%$ milk yield improvement $(21.8$ v. $20.0 \mathrm{~kg} \mathrm{FCM}$ for $H$ and $B$ rates, respectively), i.e. a mean efficiency of $0.6 \mathrm{~kg} \mathrm{FCM} / \mathrm{kg}$ supplement. This response tended to be greater in the higheryielding cows and for the high stocking rate. The sward measurements contributed to a better understanding of herbage utilization by the cows.

HODEN A., MULLER A., PEYRAUD J.L., DELABY L., FAVERDIN Ph. 1991. Pâturage pour vaches laitières. Effets du chargement et de la complémentation en pâturage tournant simplifié. INRA Prod. Anim., 4 (3), 229-239. 